\title{
Sensitivity, Equilibria, and Lyapunov Stability Analysis in Droop's Nonlinear Differential Equation System for Batch Operation Mode of Microalgae Culture Systems
}

\author{
Abraham Guzmán-Palomino ${ }^{1,+} \mathbb{D}$, Luciano Aguilera-Vázquez ${ }^{1, *}+\stackrel{\mathbb{D}}{ }$, Héctor Hernández-Escoto ${ }^{2}$ (D) \\ and Pedro Martin García-Vite ${ }^{1}$ (D) \\ 1 Tecnológico Nacional de México, Instituto Tecnológico de Ciudad Madero, Av. 1o. de Mayo esq. Sor Juana \\ Inés de la Cruz S/N Col., Los Mangos, Ciudad Madero 89440, Mexico; \\ d18073012@itcm.edu.mx (A.G.-P.); pedro.gv@cdmadero.tecnm.mx (P.M.G.-V.) \\ 2 Departamento de Ingeniería Química, Universidad de Guanajuato, Guanajuato 36000, Mexico; hhee@me.com \\ * Correspondence: Luciano.av@cdmadero.tecnm.mx; Tel.: +52-833-357-48-20 \\ + These authors contributed equally to this work.
}

check for updates

Citation: Guzmán-Palomino, A.;

Aguilera-Vázquez, L.;

Hernández-Escoto, H.;

García-Vite, P.M. Sensitivity,

Equilibria and Lyapunov Stability

Analysis in Droop's Nonlinear

Differential Equation System for

Batch Operation Mode of Microalgae

Culture Systems. Mathematics 2021, 9 ,

2192. https://doi.org/10.3390/

math9182192

Academic Editors: Pedro Beirão and Duarte Valério

Received: 20 July 2021

Accepted: 23 August 2021

Published: 8 September 2021

Publisher's Note: MDPI stays neutral with regard to jurisdictional claims in published maps and institutional affiliations.

Copyright: (c) 2021 by the authors. Licensee MDPI, Basel, Switzerland. This article is an open access article distributed under the terms and conditions of the Creative Commons Attribution (CC BY) license (https:/ / creativecommons.org/licenses/by/ $4.0 /)$.
Abstract: Microalgae-based biomass has been extensively studied because of its potential to produce several important biochemicals, such as lipids, proteins, carbohydrates, and pigments, for the manufacturing of value-added products, such as vitamins, bioactive compounds, and antioxidants, as well as for its applications in carbon dioxide sequestration, amongst others. There is also increasing interest in microalgae as renewable feedstock for biofuel production, inspiring a new focus on future biorefineries. This paper is dedicated to an in-depth analysis of the equilibria, stability, and sensitivity of a microalgal growth model developed by Droop (1974) for nutrient-limited batch cultivation. Two equilibrium points were found: the long-term biomass production equilibrium was found to be stable, whereas the equilibrium in the absence of biomass was found to be unstable. Simulations of estimated parameters and initial conditions using literature data were performed to relate the found results to a physical context. In conclusion, an examination of the found equilibria showed that the system does not have isolated fixed points but rather has an infinite number of equilibria, depending on the values of the minimal cell quota and initial conditions of the state variables of the model. The numerical solutions of the sensitivity functions indicate that the model outputs were more sensitive, in particular, to variations in the parameters of the half saturation constant and minimal cell quota than to variations in the maximum inorganic nutrient absorption rate and maximum growth rate.

Keywords: renewable energies; microalgae-batch culture; stability; sensitivity; Droop model

\section{Introduction}

Currently, about $90 \%$ of energy needs are met by burning fossil fuels such as natural gas and petroleum. With the current trends in economic globalization and energy consumption, world oil reserves may run out by 2050 [1]. Various alternative energy sources that are renewable, economically competitive, and environmentally friendly, such as geothermal, wind, solar, and bioenergy, are promising energy resources for the coming years [2]. Microalgae have been classified as third-generation biofuels and as a renewable source [3]. The microalgal biomass can be converted into energy by biological or thermo-chemical methods. Biological conversion can be performed by the fermentation of degradable components in order to produce energy compounds such as biogas, biohydrogen, bioethanol, or biobutanol, or by the extraction of oils from lipids for biodiesel production. In the environmental field, microalgae production systems mitigate the greenhouse gas emissions generated by other industrial processes, which mainly cause global warming and contribute to the creation of contaminated environments. Microalgae use an efficient biological system that is capable of growth in water and can absorb sunlight and 
capture carbon dioxide $\left(\mathrm{CO}_{2}\right)$ for the production of organic compounds with high value from photosynthesis [3]. Over the last decade, microalgae-based biomass has been globally studied because it is capable of producing several important biochemicals, such as lipids, proteins, carbohydrates, and pigments, amongst others. These biochemicals are used as feedstock in the manufacturing of value-added products for human consumption and animal feed, pharmaceutics, biofertilizers, pigments and cosmetics, and for the synthesis of antimicrobial, antiviral, and antibacterial products and medical research, to provide just a few examples from a whole spectrum of possible applications $[4,5]$. The aforementioned systems can even be used in wastewater treatment processes [6,7]. Microalgae can be grown in seawater or wastewater and do not use arable land; therefore, they do not affect agricultural land availability [8-10]. Currently, many research efforts are being devoted to the introduction of microalgae-based biomass production on a large scale [4]. Designing, monitoring, controlling, and optimizing microalgae culture systems are necessary tasks for large-scale microalgae cultivating systems [9]. Dynamic models are important tools for optimizing and controlling microalgae-based biomass systems at the laboratory and on large scales. Dynamic models might be useful to accomplish those tasks and to achieve the optimal biomass-based production of valuable metabolites. Therefore, understanding the dynamic behavior of biomass can help reduce the gap between laboratory-scale observations and those at the industrial scale [5,11,12].

In the last decade, an array of mathematical models that describe microalgal growth kinetics regarding the effect of process parameters, such as light, temperature, nutrients, oxygen accumulation, salinity, $\mathrm{pH}$, and organic or inorganic carbon, on the growth rate of microalgal cells has been presented to understand their application in large-scale microalgal production $[13,14]$. These conditions fluctuate over time regularly, and this behavior is not considered in most of the models; the effects of these variations on growth are extremely important factors to be researched for large-scale applications [14]. The existing kinetic models can be classified into three groups. The first group considers either a single internal nutrient storage factor or an external nutrient concentration factor as well as $\mathrm{N}, \mathrm{P}$, and $\mathrm{CO}_{2}$. The second group comprises models that consider a light intensity factor, and the third group considers multiple factors (e.g., both substrate and light) [13]. The models considering the external nutrient concentration assume that the growth rate depends on the nutrient concentration in culture solution. The external nutrients are easy to measure; for this reason, models in this group are widely applied. This kind of model can describe growth under low and moderate nutrient concentrations, but they are limited in terms of explaining growth under nutrient absence in growth media [13]. The Monod model is a representative model in this context that considers only nutrient limitation conditions [7]. Many researchers have applied the Monod model structure functionalities to describe the relationship between microalgae growth and a single nutrient concentration because of its simple formula. The Monod model is suitable to describe growth under low and moderate nutrient concentrations, but this model is limited in terms of describing microalgae growth inhibition due to high nutrient concentrations. In particular, the inhibition of microalgae growth occurs when concentrations of $\mathrm{NH}_{3}$ are above $300 \mathrm{mg} \mathrm{L}^{-1}$ in culture solution [15]. Modifications have been developed to improve the Monod model to overcome microalgae growth inhibition. This is the case for the Andrews model, which includes a term to describe the inhibitory effect of nutrients on the growth rate at high concentrations [16]. For example, this model was found to be preferable to the Monod model for describing the effect of $\mathrm{CO}_{2}$ concentrations on the growth of Chlorella vulgaris under $0 \%$ to $30 \% \mathrm{CO}_{2}$ enrichment of feed air, as reported by Kurano and Miyachi [17]. Growth under nutrient absence in growth media is also a weak point of the Monod model. Martínez Sancho added an additional maximum specific growth rate in the absence of an external nutrient to the Monod model; therefore, when a nutrient in the culture is exhausted, the specific growth rate is not equal to zero but to the additional maximum specific growth rate [18]. Although a better description is provided for the relationship between microalgae growth and a single nutrient, this model is not often applied due to the additional parameters that need 
to be experimentally determined. In real cultures, algae can still grow when a nutrient is absent from the growth medium because of nutrient storage in the cell $[19,20]$. The internal nutrient storage models are based on the assumption that the growth rate depends on the internal nutrient concentration in the cell, measured by the cell quota, which is the amount of intercellular nutrients per cell $[13,21]$.

The second kind of growth kinetic model considers the effect of light. These models are preferable for low and moderate algae concentrations under laboratory conditions because it is assumed that each individual cell equally receives incident light intensity, i.e., there is minimal self-shading caused by microalgae cells in the culture. For example, the Tamiya model is the most widely applied model describing the effect of light on microalgae growth: it has a simple structure and it is analogous to a Monod-type [22]. For example, Chae reported that under laboratory conditions and using fluorescent lamps, the Tamiya model was able to describe the growth of Euglena gracilis (about 0-550 $\mu$ mol photon $\mathrm{m}^{-2}$ $\mathrm{s}^{-1}$ ) with kinetic parameters of $\mu_{\max }$ as $0.06 \mathrm{~h}^{-1}$ and $K_{I}$ as $178 \mu \mathrm{mol}$ photon $\mathrm{m}^{-2} \mathrm{~s}^{-1}$ [23]. Many empirical models have been developed to describe the light-limitation effect, such as van Oorschot [24], Bannister [25], and Chalker [26].

The models considering multiple factors, such as both multiple nutrient resources and light, are based on either threshold or multiplicative theory. Although these models offer a better description for microalgae growth, they have complex model structures with many parameters, making their application impractical compared to existing experimental methods for parameter determination. An overview of such models can be found in [13]. Although many models are available for microalgae growth, it is well-known that modeling reality too closely in terms of the growth may result in an intractable model from which little can be learned at the laboratory scale. In this study, we attempted to obtain an understanding of the possible behaviors of microalgae processes considering the simplest ones, using parameters that can be easily determined by an experimental approach for batch operation systems. One of the most classical models of microalgal cultivation under substrate limitation was originally developed by Droop (1974) [27]. This model provides an empirical description of algae growth and is based on the observation of algal cultures rather than any a priori physiological hypotheses, which makes it suitable for predictive applications of microalgae growth. Droop proposed an effective relationship that estimates the specific growth rate only in terms of the cell quota [28]. This classical model uncouples substrate uptake and describes biomass growth rate as a function of this internal quota only $[27,29]$. This model has been a key to more elaborate models, including additional effects such as light photoacclimation and inhibition processes [30]. Additionally, the experimental laboratory-scale application of this model and parameters estimation have been well-reported [13,31,32]. The global stability of the Droop model was studied in continuous mode by Lange and Oyarzun $[33,34]$. They found that depending on a particular combination of the underlying parameters, there is a unique positive equilibrium that is globally asymptotically stable. Nonetheless, this work was conducted with nondimensional Droop's equations, which makes their mathematical study inapplicable to batch mode operation. Notably, it was found that chaotic dynamics do not occur in the Droop model $[33,35]$. This paper is dedicated to an in-depth analysis of the equilibria and stability behavior of the Droop model for batch mode operation culture systems based on the Lyapunov stability theory method [33,36-38]. The increasing number of applications of microalgae-based technologies encouraged our study of the main processes, factors, parameters, and variables that most influence microalgae growth in different types of cultures, including batch cultivation. A sensitivity analysis was conducted to determine how sensitive the solutions of the model are with respect to the parameters [39-41]. 


\section{Droop Kinetics-Based Model for Batch Processes}

The Droop model nonlinear differential equations for batch mode are given by Equations (1)-(3), involving three state variables: the concentration of biomass $x$, the concentration of nutrient $y$, and the intracellular quota $z$.

$$
\begin{array}{r}
\dot{x}=\mu(z) x \\
\dot{y}=-\rho(y) x \\
\dot{z}=\rho(y)-\mu(z) x
\end{array}
$$

The uptake rate $\rho(y)$ is defined by a Monod type model description, where $k_{y}$ is the half saturation constant and $\rho_{m}$ is the maximum inorganic nutrient absorption rate, as shown in Equation (4) [42,43].

$$
\rho(y)=\rho_{m} \frac{y}{y+k_{y}}
$$

The specific growth rate $\mu(z)$ is defined as a function of the intracellular quota $z, \mu_{m}$ is the maximum growth rate, and $z c_{0}$ is the minimal cell quota or minimum required quota by the organism before growth can proceed, as described by Equation (5) $[42,43]$.

$$
\mu(z)=\mu_{m}\left(1-\frac{z c_{0}}{z}\right)
$$

\section{Materials and Methods}

First, the equilibrium of the considered system was determined. As known, at an equilibrium point, the derivative is zero; then, equations are zeroed, and the resulting algebraic equations are solved for the states variables $x, y$, and $z$. The Lyapunov stability theory was used to describe the stability of the considered dynamic system $[34,38,40,41,44-46]$. Simulations of algae growth were used to illustrate the results of the stability analysis. Then, a sensitivity analysis was performed through solving the sensitivity functions at nominal values to provide first-order estimates of the effect of the model parameters variations on solutions over time. The simulations of the obtained sensitivity equations were computed with nominal parameters based on the literature [11,39,40,44]. The Livermore Solver for Ordinary Differential Equations (LSODE) function was used to numerically solve the ordinary differential equation systems; the methods included therein for the numerical solution can be found in [47].

\section{Results}

Two equilibria were found at the stationary state of the considered model. The equilibrium points represent either long-term survival for the organism or the instant just before starting the biomass inoculation in a batch mode culture system. Both cases are given by:

$$
\bar{v}_{1}=\left(c_{1}, 0, z c_{0}\right)
$$

and

$$
\bar{v}_{2}=\left(0,-\frac{c_{2} k_{y} \mu_{m}}{-z c_{0} \mu_{m}+c_{2} \mu_{m}-\rho_{m}}, c_{2}\right)
$$

To determine the stability of the equilibrium points $\bar{v}_{1}$ and $\bar{v}_{2}$, the Jacobian matrix of partial derivatives of the considered system is provided in Equation (8): 


$$
D f=\left(\begin{array}{ccc}
\mu(z) & 0 & \frac{z c_{0} \mu_{m} x}{z^{2}} \\
-\rho(y) & \frac{\rho(y) x}{\left(y+k_{y}\right)}-\frac{\rho_{m} x}{y+k_{y}} & 0 \\
0 & \frac{\rho_{m}}{y+k_{y}}-\frac{\rho(y)}{\left(y+k_{y}\right)} & -\mu(z)-\frac{z c_{0} \mu_{m}}{z}
\end{array}\right)
$$

Evaluating $D f$ at $\bar{v}_{1}=\left(c_{1}, 0, z c_{0}\right)$ yields:

$$
D f_{\left(\bar{v}_{1}\right)}=\left(\begin{array}{ccc}
0 & 0 & \frac{c_{1} \mu_{m}}{z c_{0}} \\
0 & \frac{c_{1} \rho_{m}}{k_{\delta_{m}}} & 0 \\
0 & -\frac{\rho_{m}}{k_{s}} & -\mu_{m}
\end{array}\right)
$$

whereas evaluating $D f$ at $\bar{v}_{2}$ yields:

$$
\begin{aligned}
& D f_{\left(\bar{v}_{2}\right)}=\left[\begin{array}{lll}
\vec{A} \mid & \vec{B} & \vec{C}
\end{array}\right] \\
& \vec{A}=\left[\begin{array}{c}
\left(1-\frac{z c_{0}}{c_{2}}\right) \mu_{m} \\
\frac{c_{2} k_{y}{ }^{2} \rho_{m} \mu_{m}^{2}-z c_{0}}{\left(c_{2} \mu_{m} \rho_{m}-z c_{0} \mu_{m}\right)\left(k_{y}-\frac{c_{2} k_{y}{ }^{2} \mu_{m}^{2}-z c_{0}}{c_{2} \mu_{m}-\rho_{m}-z c_{0} \mu_{m}}\right)} \\
0
\end{array}\right] \\
& \vec{B}=\left[\begin{array}{c}
0 \\
\frac{\rho_{m}}{\rho_{y}-\frac{c_{2} k_{y}^{2} \mu_{m}^{2}-z c_{0}}{c_{2} \mu_{m}-\rho_{m}-z c_{0} \mu_{m}}}+\frac{c_{2} k_{y}{ }^{2} \rho_{m} \mu_{m}^{2}-z c_{0}}{\left(c_{2} \mu_{m} \rho_{m}-z c_{0} \mu_{m}\right)\left(k_{y}-\frac{c_{2} k_{y}{ }^{2} \mu_{m}^{2}-z c_{0}}{c_{2} \mu_{m}-\rho_{m}-z c_{0} \mu_{m}}\right)^{2}}
\end{array}\right] \\
& \vec{C}=\left[\begin{array}{c}
0 \\
0 \\
\left(1-\frac{z c_{0}}{c_{2}}\right) \mu_{m}-\frac{z c_{0} \mu_{m}}{c_{2}}
\end{array}\right]
\end{aligned}
$$

The eigenvalues obtained of $D f\left(\bar{v}_{1}\right)$ and $D f\left(\bar{v}_{2}\right)$ are provided by Equations (14) and (15):

$$
D f\left(\bar{v}_{1}\right)=\left(-\frac{c_{1} \rho_{m}}{k_{y}},-\mu_{m}, 0\right)
$$




$$
\operatorname{Df}\left(\bar{v}_{2}\right)=\left(-\frac{\left(z c_{0}-c_{2}\right) \mu_{m}}{c_{2}},-\mu_{m}, 0\right)
$$

As can be seen, both eigenvalues have nonpositive real parts and there is at least one eigenvalue with a zero real part. Therefore, it is not sufficient to determine the stability of the point on the basis of the eigenvalues $D f\left(\bar{v}_{1}\right)$ and $D f\left(\bar{v}_{2}\right)$. Instead, it was necessary to examine the model equations as follows. First, it is important to demarcate the physical meaning of the found equilibria. In the case of the equilibrium point $\bar{v}_{1}=\left(c_{1}, 0, z c_{0}\right)$, from a physical sense, the existence of biomass $x=c_{1}$ in the microalgae growth system when the external nutrient uptake is entirely consumed means $y=0$; thus, the intracellular quota $z$ reaches a minimal cell quota $z c_{0}$, as shown in Figure 1.

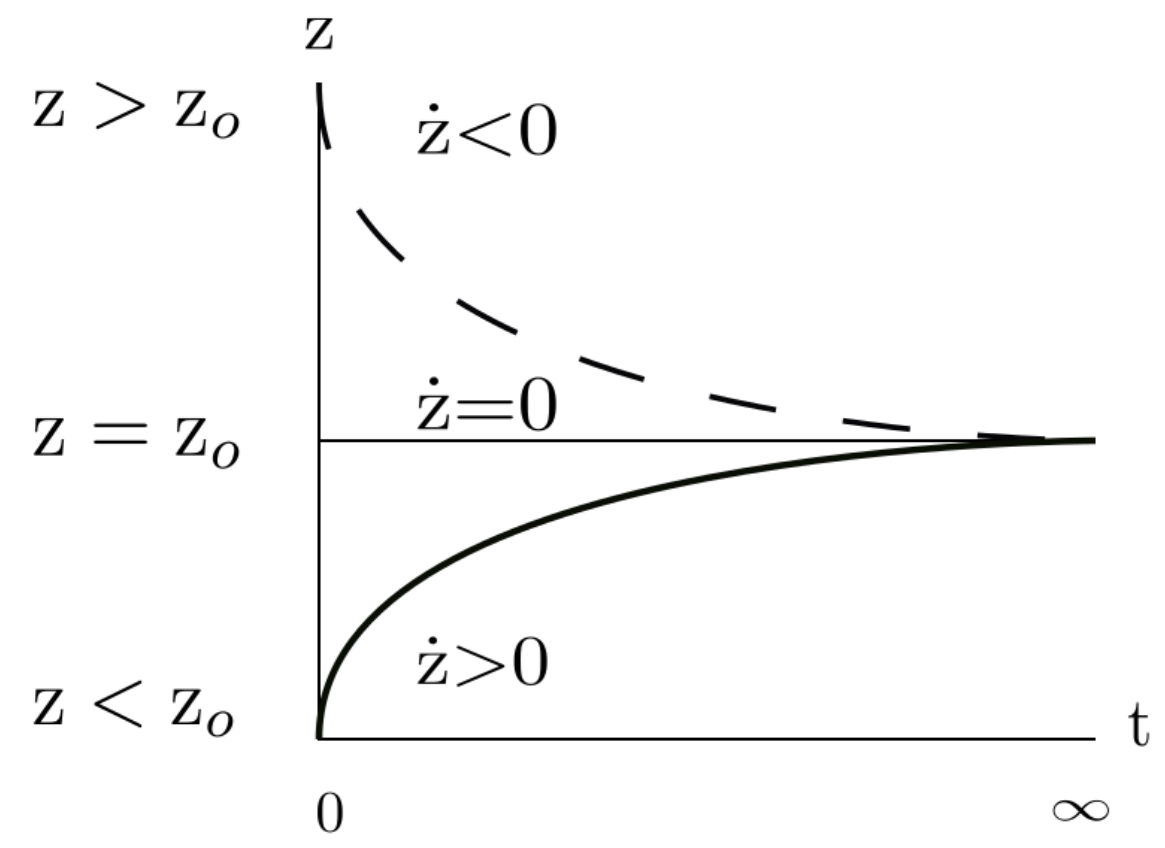

Figure 1. Behavior of intracellular quota $z$ over time under three conditions of the state variable. According to the definition of $z c_{0}, z$ must be greater than $z c_{0}$ to have biological meaning. Note that when $z>z c_{0}, \dot{z}$ is negative. This means that during that time, $z$ decrease, and for $z<z c_{0}, \dot{z}$ is positive, which implies that the minimum concentration of the limiting nutrient per cell required before growth can proceed, is trying to be reached. Therefore, during that time, there is no biomass production until $z=z c_{0}$ is reached. However, when $z=z c_{0}$, biomass production is evidently available. Note that $\lim _{y \rightarrow \infty}=0$ at $\overline{v_{1}}$, which implies that $\lim _{z \rightarrow \infty}=z c_{0}$ for all $t>0$ in batch mode cultures.

The equilibrium point:

$$
\bar{v}_{2}=\left(0,-\frac{c_{2} k_{y} \mu_{m}}{-z c_{0} \mu_{m}+c_{2} \mu_{m}-\rho_{m}}, c_{2}\right)
$$

from a physical sense means the absence of biomass. As such, let us begin with the equilibria stability analysis.

\subsection{Finding Stability in Long-Term Biomass Production Equilibrium}

For the equilibrium $\bar{v}_{1}$, it can be observed that the real part $-c_{1} \rho_{m} / k_{y}$ of the eigenvalue $D f\left(\bar{v}_{1}\right)$ involves the values of the parameters of the maximum absorption rate, half saturation constant, and the state variable of biomass, $x=c_{1}$. Microalgae growth systems do not admit negative values for these quantities; therefore, this real part of the eigenvalue 
$D f\left(\bar{v}_{1}\right)$ always remains negative. For the real part, similarly, $-\rho_{m}$ of $D f\left(\bar{v}_{1}\right)$, which is the maximum absorption rate, and neither admits negative values from a physical point of view. Note also that the value of $x=c_{1}$, where $c_{1}$ is an undefined constant, implies that biomass can take a value of an infinite possible sets of values at the stationary state $(\Omega$ set). Hence, the system does not have only one equilibrium rather, it has an entire line of equilibria, that is, the considered nonlinear system can have infinite equilibria.

To explore another alternative to prove the stability of $\bar{v}_{1}$, the equations of the system (1), (2) and (3) can be easily reduced to a two-dimensional system as follows:

Solving for the expressions $\frac{\dot{x}}{x}$ and $\frac{\dot{y}}{x}$ from Equations (1) and (2), respectively, yields:

$$
\begin{aligned}
\frac{\dot{x}}{x} & =\mu_{m}\left(1-\frac{z c_{0}}{z}\right) \\
\frac{\dot{y}}{x} & =-\rho_{m} \frac{y}{y+k_{y}}
\end{aligned}
$$

These expressions are then substituted in Equation (3):

$$
\dot{z}=\frac{-\dot{y}-\dot{x} z}{x}
$$

Then, the term $-\dot{y}$ can be solved from Equation (19):

$$
-\dot{y}=\dot{x} z+x \dot{z}
$$

By integrating both sides of Equation (20), the concentration of nutrient $y$ is obtained:

$$
\begin{gathered}
-\int \dot{y}=\int(\dot{x} z+x \dot{z}) \\
\alpha-y=x z
\end{gathered}
$$

Finally, the value of the concentration of nutrient $y$ is given in Equation (23):

$$
y=\alpha-x z
$$

Therefore, $y$ is not independent of $x$ and $z$, whereas $\alpha=x(t) y(t)+z(t)$ for all $t \geq 0$, with $\alpha$ defined as $\alpha=x_{0} y_{0}+z_{0}$, where $x_{0}, y_{0}$, and $z_{0}$ are the initial conditions.

Substituting $y$ from Equation (23) into Equations (1) and (3), and discarding Equation (2), results in:

$$
\begin{gathered}
\dot{x}=\mu_{m}\left(1-\frac{z c_{0}}{z}\right) x \\
\dot{z}=\rho_{m} \frac{\alpha-x z}{\alpha-x z+k_{y}}-\mu_{m}\left(1-\frac{z c_{0}}{z}\right)
\end{gathered}
$$

Equations (24) and (25) describe a two-dimensional system in terms of $x$ and $z$. The analysis of such a system should lead to the description of the local stability of the system.

Two equilibria were found at the stationary state of the new two-dimensional system. The equilibria $\bar{v}_{3}$ and $\bar{v}_{4}$ represent either long-term survival of the organism or the absence of biomass, respectively, in a batch mode culture system, which are given by:

$$
\bar{v}_{3}=\left(\frac{\alpha}{z c_{0}}, z c_{0}\right)
$$

and

$$
\bar{v}_{4}=\left(0,-\frac{\left(k_{y}+\alpha\right) \mu_{m} z c_{0}+\alpha \rho_{m}}{\left(k_{y}+\alpha\right) \mu_{m}}\right)
$$

The Jacobian matrix of the reduced system, described by Equations (24) and (25), is: 


$$
D f_{2}=\left[\begin{array}{ll}
\vec{D} \mid & \vec{E}
\end{array}\right]
$$

where

$$
\begin{gathered}
\vec{D}=\left[\begin{array}{c}
\mu(z) \\
-{\frac{\rho_{m} z(\alpha-x z)^{2}}{-x z+k_{y}+\alpha}}^{2}-\frac{\rho_{m} z}{-x z+k_{y}+\alpha}
\end{array}\right] \\
\vec{E}=\left[\begin{array}{c}
\frac{\mu_{m} x z c_{0}}{z^{2}} \\
-\mu(z)-\frac{\mu_{m} z c_{0}}{z}-\frac{\rho_{m} x}{-x z+k_{y}+\alpha}+\frac{\rho_{m} x(\alpha-x z)^{2}}{-x z+k_{y}+\alpha}
\end{array}\right]
\end{gathered}
$$

Evaluating $\bar{v}_{3}$ in $D f_{2}$ :

$$
D f_{2}\left(\bar{v}_{3}\right)=\left[\begin{array}{ll}
\vec{D}_{a} & \vec{E}_{a}
\end{array}\right]
$$

where

$$
\begin{gathered}
\vec{D}_{a}=\left[\begin{array}{c}
0 \\
-\frac{\rho_{m} z_{0}}{k_{y}}
\end{array}\right] \\
\vec{E}_{a}=\left[\begin{array}{c}
\frac{\alpha \mu_{m}}{z c_{0}^{2}} \\
\frac{\alpha \rho_{m}}{k_{y} z c_{0}}-\mu_{m}
\end{array}\right]
\end{gathered}
$$

The real parts of the eigenvalues obtained from the evaluated $D f_{2}$, Equation (28) at $\bar{v}_{3}$, and Equation (26), are:

$$
D f_{2}\left(\bar{v}_{3}\right)=\left(-\frac{\alpha \rho_{m}}{k_{y} z c_{0}},-\mu_{m}\right)
$$

Since the real part of each eigenvalue of $D f_{2}\left(\bar{v}_{3}\right)$ is negative, then $\bar{v}_{3}$ is stable for all $x_{0}>0, y_{0}>0$ and $z_{0}>0$.

\subsection{Finding the Stability of the Absence of Biomass Equilibrium}

For the equilibrium of $\bar{v}_{2}$, it is possible to observe the real part

$$
\bar{v}_{2}=-\frac{\left(z c_{0}-c_{2}\right) \mu_{m}}{c_{2}}
$$

of the eigenvalue $D f_{\overline{v_{2}}}$, where $c_{2}=z$ at the stationary state, and for $z>z c_{0}$, the sign remains positive; thus, the equilibrium $\overline{v_{2}}$ is unstable. Conversely, if $z<z c_{0}$, the equilibrium $\overline{v_{2}}$ is stable, as shown in Figure 1. The latter condition is physically meaningless for microalgae growth systems because if $\bar{v}_{2}$ is stable, the microalgae growth system would always trend to the absence of biomass, but this does not happen. Rather, microalgae cultures trend to growth under real conditions for initial values of $x_{0} \neq 0$. 
If the two-dimensional system aforementioned is considered to evaluate the stability of the equilibrium of the absence of biomass, it yields:

Evaluating $\bar{v}_{4}$, Equation (27) in $D f_{2}$, and Equation (28) yields:

$$
D f_{2}\left(\bar{v}_{4}\right)=\left[\begin{array}{ll}
\vec{F}_{a} \mid & \vec{G}_{a}
\end{array}\right]
$$

where:

$$
\begin{gathered}
\vec{F}_{a}=\left[\begin{array}{c}
\mu_{m}\left(1-\frac{\left(k_{y}+\alpha\right) \mu_{m} z c_{0}}{\left(k_{y}+\alpha\right) \mu_{m} z c_{0}+\alpha \rho_{m}}\right) \\
\frac{\alpha \rho_{m}\left(\left(k_{y}+\alpha\right) \mu_{m} z c_{0}+\alpha \rho_{m}\right)}{\left(k_{y}+\alpha\right)^{3} \mu_{m}}
\end{array}\right] \\
\vec{G}_{a}=\left[\begin{array}{c}
0 \\
-\mu_{m}\left(1-\frac{\left(k_{y}+\alpha\right) \mu_{m} z c_{0}}{\left(k_{y}+\alpha\right) \mu_{m} z c_{0}+\alpha \rho_{m}}\right)-\frac{\left(k_{y}+\alpha\right) \mu_{m}{ }^{2} z c_{0}}{\left(k_{y}+\alpha\right) \mu_{m} z c_{0}+\alpha \rho_{m}}
\end{array}\right]
\end{gathered}
$$

The real parts of the eigenvalues obtained from the evaluated $D f_{2}$ Equation (28) at $\bar{v}_{4}$ and Equation (27) are:

$$
D f_{2}\left(\bar{v}_{4}\right)=\left(\frac{\alpha \rho_{m} \mu_{m}}{\left(k_{y}+\alpha\right) \mu_{m} z c_{0}+\alpha \rho_{m}},-\mu_{m}\right)
$$

Since the real part of at least one eigenvalue of $D f_{2}\left(\bar{v}_{4}\right)$ is positive, $\bar{v}_{4}$ is unstable.

To represent the stability of the equilibrium point $\bar{v}_{1}$, a bifurcation diagram was used, as shown in Figure 2. These equilibria only depend on the value of initial conditions. Notably, the value of $\bar{v}_{1}$ only includes the $z c_{0}$ parameter for the value of $z$ in the stationary state, which means that changes in this parameter result in a change in the equilibrium point $\bar{v}_{1}$ of the system and, particularly of interest, in the amount of biomass (Figure 3). The parameters and initial conditions were obtained from [48]. Additionally, the bifurcation diagram of the state variable $y$ is illustrated in Figure 4.

The diagram in Figure 2 shows that the stable steady state $\bar{v}_{1}$ is stable for $z c_{0}>0$ parameter values and unstable for $z c_{0}<0$ values. As can be observed, for practical applications, it does not have significance. The $\Omega$ sets collide and exchange stability at $z=0$. The stability of $\overline{v_{1}}$ depends on the value of the minimal cell quota parameter at a given initial condition. According to the above description, $\overline{v_{1}}$ is stable. 


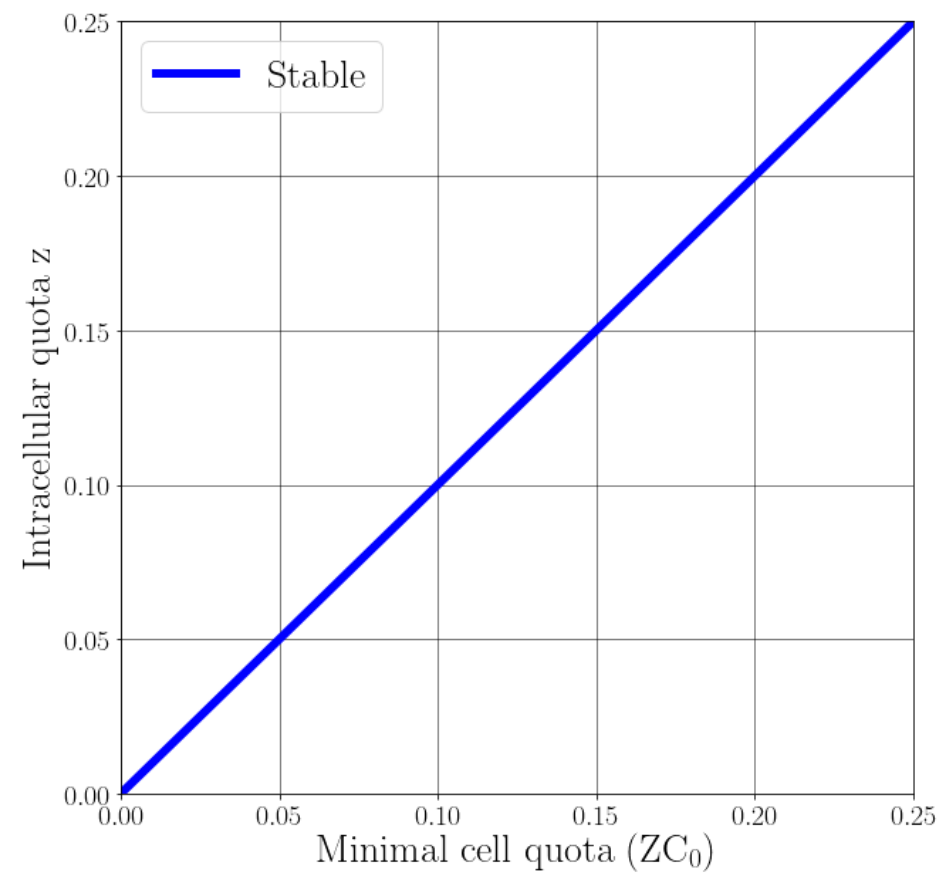

Figure 2. A bifurcation diagram of the intracellular quota $z$ vs. minimal cell quota $z c_{0}$. For each value of $z c_{0}$, the diagram shows the corresponding equilibrium points. We prepared the plot in Python 3.7.1.

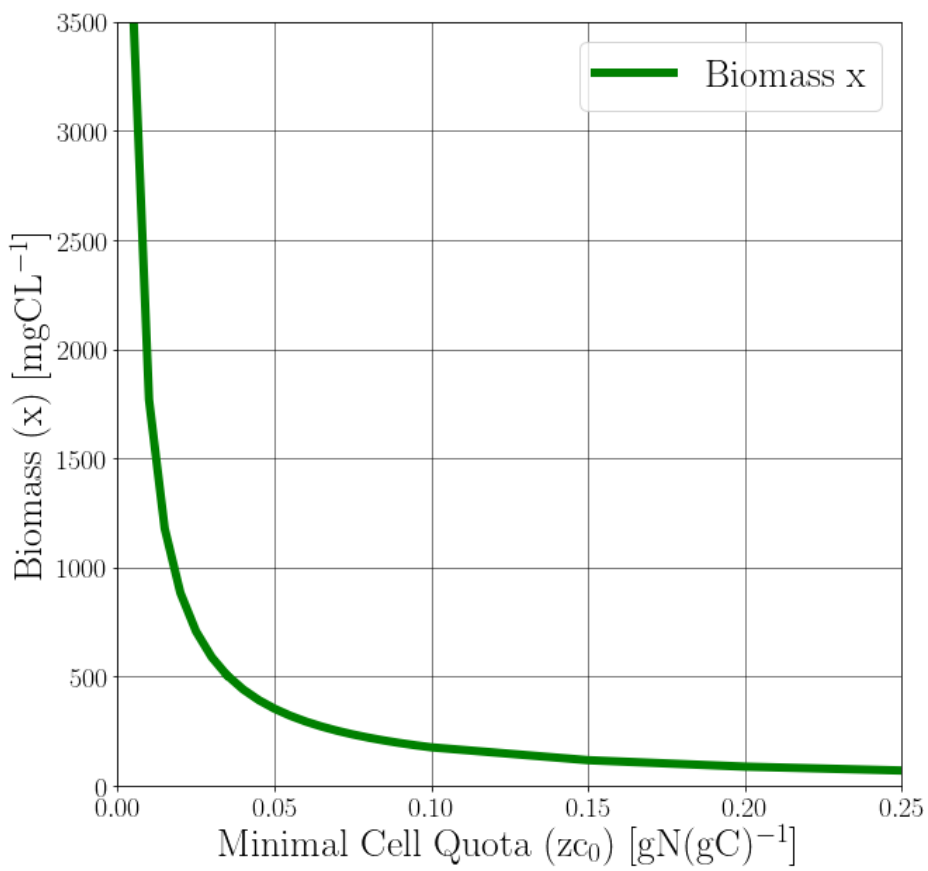

Figure 3. A bifurcation diagram of the biomass $(x)$ vs. minimal cell quota $\left(z c_{0}\right)$. The horizontal axis of this figure indicates the values of th minimal cell quota $z c_{0}$, and the vertical axis shows the values of biomass $x$. For each value of $z c_{0}$, the diagram shows the corresponding equilibrium points. Parameters: $\rho_{m}=0.0916 \mathrm{mgN}(\mathrm{mgC})^{-1} \mathrm{~d}^{-1}, k_{s}=1.7499 \mathrm{mgNL}^{-1}, \mu_{m}=1.8102 \mathrm{~d}^{-1}$, and $z c_{0}$ was varied from 0.01 to 0.25 . Initial conditions correspond to $x_{0}=91.483 \mathrm{mgCL}^{-1}, y=14.5148 \mathrm{mgNL}^{-1}$, $z_{0}=0.0347 \mathrm{mgN}(\mathrm{mgC})^{-1}$. Parameter data were obtained from [48]. We performed the simulation and plotting in Octave 5.1.0.0 and Python 3.7.1. 


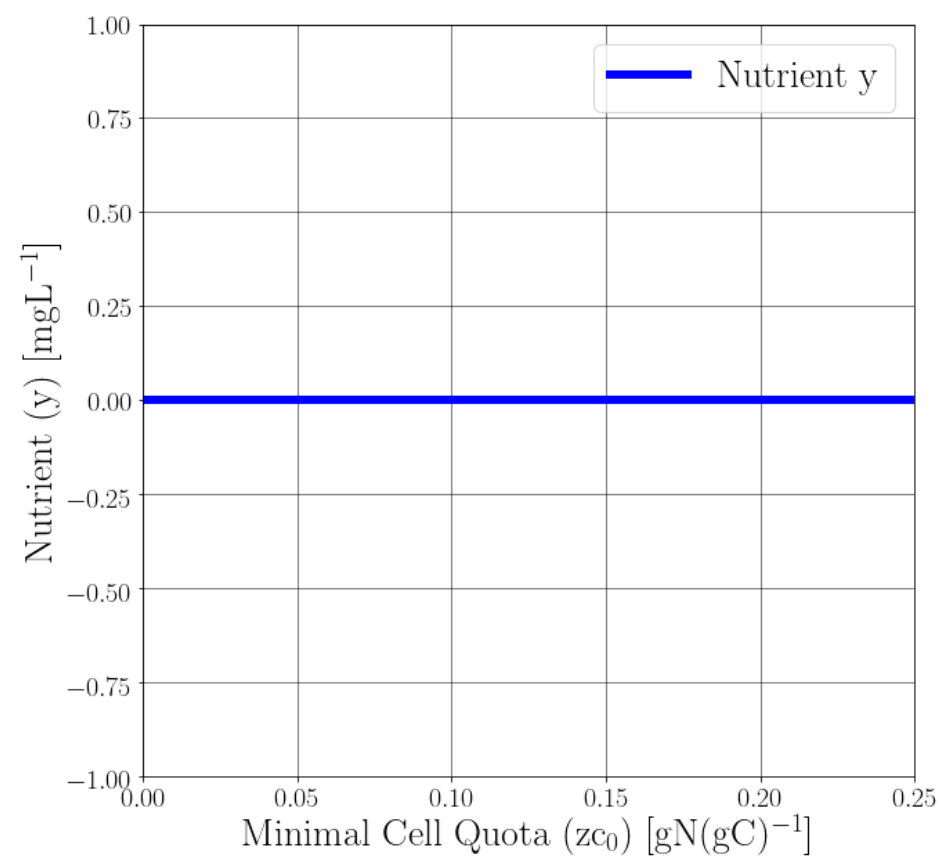

Figure 4. A bifurcation diagram of the nutrient $(y)$ vs. minimal cell quota $\left(z c_{0}\right)$. The horizontal axis of this figure indicates the values of the minimal cell quota $z c_{0}$, and the vertical axis indicates the nutrient $y$ values. For each value of $z c_{0}$, the diagram shows the corresponding equilibrium points. Parameters: $\rho_{m}=0.0916 \mathrm{mgN}(\mathrm{mgC})^{-1} \mathrm{~d}^{-1}, k_{s}=1.7499 \mathrm{mgNL}^{-1}, \mu_{m}=1.8102 \mathrm{~d}^{-1}$, and $z c_{0}$ was varied between 0.01 to 0.25 . Initial conditions correspond to $x_{0}=91.483 \mathrm{mgCL}^{-1}$, $y=14.5148 \mathrm{mgNL}^{-1}, z_{0}=0.0347 \mathrm{mgN}(\mathrm{mgC})^{-1}$. Parameter data were obtained from [48]. We performed simulation and plotting in Octave 5.1.0.0 and Python 3.7.1.

The phase space in Figure 5 illustrates that the equilibria of trajectories A and B, corresponding to the simulations in Figures 6 and 7, respectively, converge to a straight line placed on the $X Z$-plane for a given $z c_{0}$ parameter value and initial conditions.

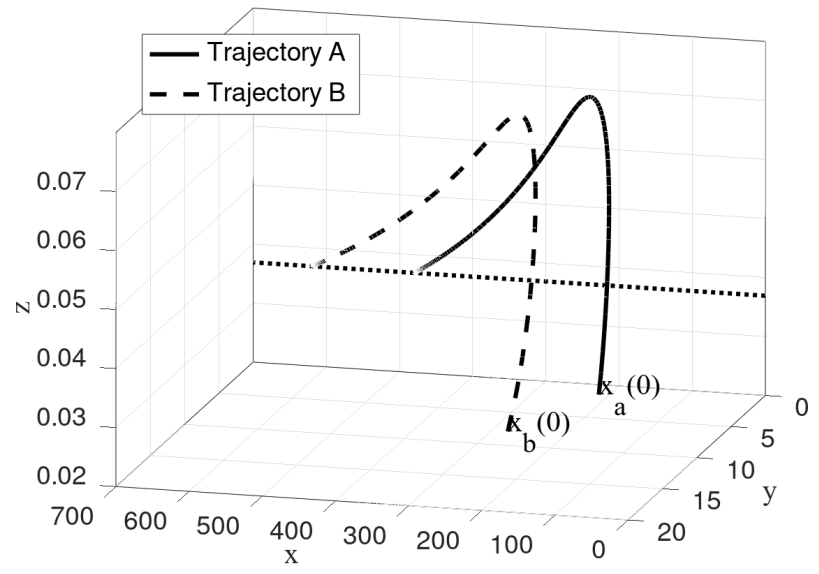

Figure 5. Phase diagram showing two trajectories resulting from simulations of the Droop model with two different initial conditions $x_{a}(0)$, and $x_{b}(0)$, shown in Figures 6 and 7 . The parameters and initial conditions are shown in Figures 6 and 7. Note that both trajectories A and B are attracted to a stable region (dotted line) on the XZ-plane when $z$ tends to the parameter $z c_{0}$. We performed the simulation and plotting in Octave 5.1.0.0. 


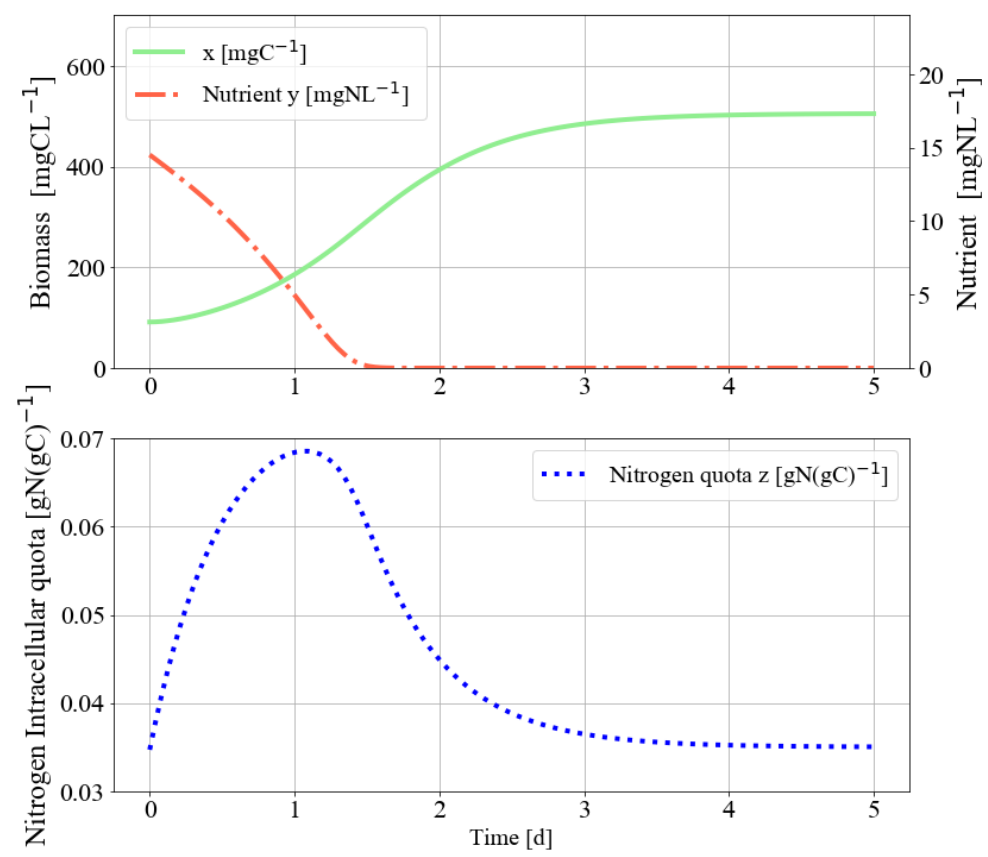

Figure 6. Batch mode simulation of biomass $x$, limiting nutrient $y$, and intracellular quota $z$ concentrations vs. cultivation time under a constant light intensity of $150 \mu \mathrm{molm}^{-2} \mathrm{~s}^{-1}$, showing the final biomass production at nutrient depletion. The initial conditions correspond to $x_{0}=91.483 \mathrm{mgCL}^{-1}$, $y=14.5148 \mathrm{mgNL}^{-1}$, and $z_{0}=0.0347 \mathrm{mgN}(\mathrm{mgC})^{-1}$. The model input parameters for simulation are $\rho_{m}=0.0901 \mathrm{mgN}(\mathrm{mgC})^{-1} \mathrm{~d}^{-1}, k_{s}=1.6499 \mathrm{mgNL}^{-1}, \mu_{m}=1.9054 \mathrm{~d}^{-1}$, and $z c_{0}=0.0350 \mathrm{mgN}(\mathrm{mgC})^{-1}$. Taken from [48].
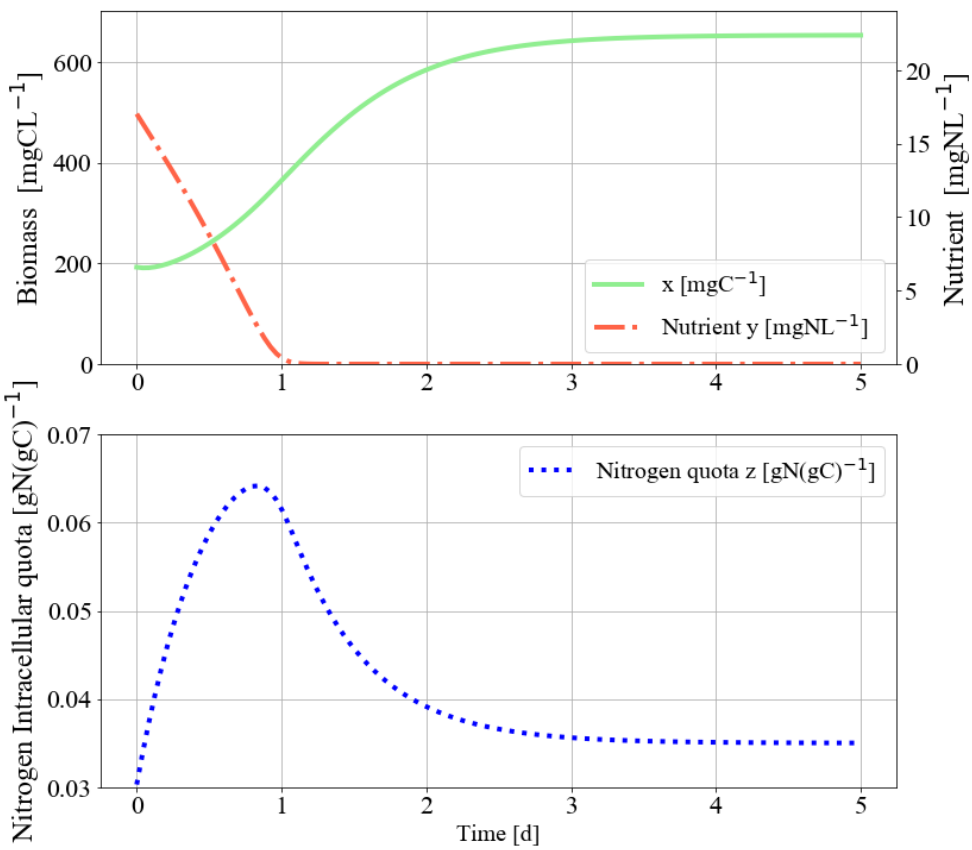

Figure 7. Batch mode simulation of biomass $x$, limiting nutrient $y$, and intracellular quota $z$ concentrations vs. cultivation time under a constant light intensity of $150 \mu \mathrm{molm}^{-2} \mathrm{~s}^{-1}$, showing the final biomass production at nutrient depletion. The initial conditions correspond to $x_{0}=192.332 \mathrm{mgCL}^{-1}, y=17.03 \mathrm{mgNL}^{-1}$, and $z_{0}=0.0303 \mathrm{mgN}(\mathrm{mgC})^{-1}$. The model input parameters for simulation are $\rho_{m}=0.0901 \mathrm{mgN}(\mathrm{mgC})^{-1} \mathrm{~d}^{-1}, k_{s}=1.6499 \mathrm{mgNL}^{-1}, \mu_{m}=1.9054 \mathrm{~d}^{-1}$, and $z c_{0}=0.0350 \mathrm{mgN}(\mathrm{mgC})^{-1}$. Taken from [48]. 


\subsection{Simulations of the Model}

The estimated parameters of the batch microalgae growth experiments were obtained from the literature to simulate microalgal growth and to observe, from a physical point of view, the behavior of the equilibria. The estimated growth parameters for the two predictions were the same except for the initial conditions. The experimental setup and parameter estimation can be found in [48]. It is important to remark upon how the equilibria changed under initial conditions, although the same parameters were used (Figures 6 and 7).

\section{Sensitivity Equations of the Droop Model in Batch Mode}

Sensitivity analysis is an important tool during model development, calibration, and optimization. Sensitivity analysis is used to identify the parameters that most influence the results obtained by the models. A sensitivity analysis of the model studied in this work helped us to better understand the interactions among inputs parameters, state variables, and output results of the predictions. It is also an important tool for building more elaborate models. Sensitivity analysis was applied to determine the sensitivities of the state variables $x, y$, and $z$ with respect to all parameters: maximum inorganic nutrients $\rho_{m}$, half saturation constant $k_{y}$, maximum growth rate $\mu$, and the minimal cell quota $z c_{0}$. Before beginning the procedure for calculating the sensitivity, it is convenient to rename the variables and parameters in Equations (1)-(3). Perhaps, the simplest approach is to define such variables as follows:

$$
x=\left[\begin{array}{c}
\dot{x}_{1} \\
\dot{x}_{2} \\
\dot{x}_{3}
\end{array}\right]=\left[\begin{array}{c}
\dot{x} \\
\dot{y} \\
\dot{z}
\end{array}\right]
$$

Let us represent the parameters as $\lambda$ and the nominal values of parameters as $\lambda_{0}$, as follows:

$$
\begin{gathered}
\lambda=\left[\begin{array}{l}
\lambda_{1} \\
\lambda_{2} \\
\lambda_{3} \\
\lambda_{4}
\end{array}\right]=\left[\begin{array}{c}
\rho_{m} \\
k_{y} \\
\mu \\
z c_{0}
\end{array}\right] \\
\lambda_{0}=\left[\begin{array}{c}
\lambda_{0_{1}} \\
\lambda_{0_{2}} \\
\lambda_{3} \\
\lambda_{0_{4}}
\end{array}\right]=\left.\left[\begin{array}{c}
\rho_{m} \\
k_{y} \\
\mu \\
z c_{0}
\end{array}\right]\right|_{\text {nominal }}
\end{gathered}
$$

Then, the nominal system is given by:

$$
\begin{array}{ll}
\dot{x}_{1}= & \lambda_{0_{3}}\left(1-\frac{\lambda_{0_{4}}}{x_{3}}\right) x_{1} \\
\dot{x}_{2}= & -\lambda_{0_{1}} \frac{x_{2}}{x_{2}+\lambda_{0_{2}}} x_{1} \\
\dot{x}_{3}= & \lambda_{0_{1}} \frac{x_{2}}{x_{2}+\lambda_{0_{2}}}-\lambda_{0_{3}}\left(1-\frac{\lambda_{0_{4}}}{x_{3}}\right) x_{3}
\end{array}
$$

The sensitivity function $\dot{S}(t)$ provides a first-order estimate of the effect of parameter variations solutions [40].

$$
\dot{S}(t)=A\left(t, \lambda_{0}\right) S(t)+B\left(t, \lambda_{0}\right), S\left(t_{0}\right)
$$


where:

$$
\begin{aligned}
& A\left(t, \lambda_{0}\right)=\left.\frac{\partial f(t, x, \lambda)}{\partial x}\right|_{x=x\left(t, \lambda_{0}\right), \lambda=\lambda_{0}} \\
& B\left(t, \lambda_{0}\right)=\left.\frac{\partial f(t, x, \lambda)}{\partial \lambda}\right|_{x=x\left(t, \lambda_{0}\right), \lambda=\lambda_{0}}
\end{aligned}
$$

The sensitivity function can be calculated by solving numerically:

$$
\begin{aligned}
& \dot{x}_{1}=f(t, x, \lambda) \quad x\left(t_{0}\right)=x_{0} \\
& \dot{x}_{\lambda}=\left[\frac{\partial f(t, x, \lambda)}{\partial x}\right] x_{\lambda}+\left[\frac{\partial f(t, x, \lambda)}{\partial \lambda}\right] \quad x \lambda\left(t_{0}\right)=0
\end{aligned}
$$

The Jacobian matrices $\frac{\partial f}{\partial x}$ and $\frac{\partial f}{\partial \lambda}$ are given by:

$$
\frac{\partial f}{\partial x}=\left(\begin{array}{ccc}
\lambda_{3}\left(1-\frac{\lambda_{4}}{x_{3}}\right) & 0 & \frac{\lambda_{4} \lambda_{3} x_{1}}{x_{3}^{2}} \\
-\frac{\lambda_{1} x_{2}}{x_{2}+\lambda_{2}} & \frac{\lambda_{1} x_{1} x_{2}}{\left(x_{2}+\lambda_{2}\right)^{2}}-\frac{\lambda_{1} x_{1}}{x_{2}+\lambda_{2}} & 0 \\
0 & \frac{\lambda_{1}}{x_{2}+\lambda_{2}}-\frac{\lambda_{1} x_{2}}{\left(x_{2}+\lambda_{2}\right)^{2}} & -\lambda_{3}\left(1-\frac{\lambda_{4}}{x_{3}}\right)-\frac{\lambda_{4} \lambda_{3}}{x_{3}}
\end{array}\right)
$$

and

$$
\frac{\partial f}{\partial \lambda}=\left(\begin{array}{cccc}
0 & 0 & x_{1}\left(1-\frac{\lambda_{4}}{x_{3}}\right) & -\frac{\lambda_{3} x_{1}}{x_{3}} \\
-\frac{x_{1} x_{2}}{x_{2}+\lambda_{2}} & \frac{\lambda_{1} x_{1} x_{2}}{\left(x_{2}+\lambda_{2}\right)^{2}} & 0 & 0 \\
\frac{x_{2}}{x_{2}+\lambda_{2}} & \frac{-\lambda_{1} x_{2}}{\left(x_{2}+\lambda_{2}\right)^{2}} & -x_{3}\left(1-\frac{\lambda_{4}}{x_{3}}\right) & \lambda_{3}
\end{array}\right)
$$

Evaluating these Jacobian matrices at the nominal parameters provided in Equation (41) yields:

$$
\left.\frac{\partial f}{\partial x}\right|_{\text {nominal }}=\left(\begin{array}{ccc}
\lambda_{0_{3}}\left(1-\frac{\lambda_{0_{4}}}{x_{3}}\right) & 0 & \frac{\lambda_{0_{4}} \lambda_{0_{3}} x_{1}}{x_{3}^{2}} \\
-\frac{\lambda_{0_{1}} x_{2}}{x_{2}+\lambda_{0_{2}}} & \frac{\lambda_{0_{1}} x_{1} x_{2}}{\left(x_{2}+\lambda_{0_{2}}\right)^{2}}-\frac{\lambda_{0_{1}} x_{1}}{x_{2}+\lambda_{0_{2}}} & 0 \\
0 & \frac{\lambda_{0_{1}}}{x_{2}+\lambda_{0_{2}}}-\frac{\lambda_{0_{1}} x_{2}}{\left(x_{2}+\lambda_{0_{2}}\right)^{2}} & -\lambda_{0_{3}}\left(1-\frac{\lambda_{0_{4}}}{x_{3}}\right)-\frac{\lambda_{0_{4}} \lambda_{0_{3}}}{x_{3}}
\end{array}\right)
$$

and

$$
\left.\frac{\partial f}{\partial \lambda}\right|_{\text {nominal }}=\left(\begin{array}{cccc}
0 & 0 & x_{1}\left(1-\frac{\lambda_{0_{4}}}{x_{3}}\right) & -\frac{\lambda_{0_{3}} x_{1}}{x_{3}} \\
-\frac{x_{1} x_{2}}{x_{2}+\lambda_{0_{2}}} & \frac{\lambda_{0_{1}} x_{1} x_{2}}{\left(x_{2}+\lambda_{0_{2}}\right)^{2}} & 0 & 0 \\
\frac{x_{2}}{x_{2}+\lambda_{0_{2}}} & \frac{-\lambda_{0_{1}} x_{2}}{\left(x_{2}+\lambda_{0_{2}}\right)^{2}} & -x_{3}\left(1-\frac{\lambda_{0_{4}}}{x_{3}}\right) & \lambda_{0_{3}}
\end{array}\right)
$$

To numerically find $S(t)$, we solve (47) for nominal values of the parameters and let $S$ be: 


$$
S=\left[\begin{array}{llll}
x_{4} & x_{7} & x_{10} & x_{13} \\
x_{5} & x_{8} & x_{11} & x_{14} \\
x_{6} & x_{9} & x_{12} & x_{15}
\end{array}\right]=\left.\left[\begin{array}{llll}
\frac{\partial x_{1}}{\partial \lambda_{1}} & \frac{\partial x_{1}}{\partial \lambda_{2}} & \frac{\partial x_{1}}{\partial \lambda_{3}} & \frac{\partial x_{1}}{\partial \lambda_{4}} \\
\frac{\partial x_{2}}{\partial \lambda_{1}} & \frac{\partial x_{2}}{\partial \lambda_{2}} & \frac{\partial x_{2}}{\partial \lambda_{3}} & \frac{\partial x_{2}}{\partial \lambda_{4}} \\
\frac{\partial x_{3}}{\partial \lambda_{1}} & \frac{\partial x_{3}}{\partial \lambda_{2}} & \frac{\partial x_{3}}{\partial \lambda_{3}} & \frac{\partial x_{3}}{\partial \lambda_{4}}
\end{array}\right]\right|_{\text {nominal }}
$$

Then, the sensitivity functions from solving (47) are described by:

$$
\begin{aligned}
& \dot{x}_{1}=\quad \lambda_{0_{3}}\left(1-\frac{\lambda_{0_{4}}}{x_{3}}\right) x_{1} \quad x_{1}(0)=x_{1_{0}} \\
& \dot{x}_{2}=x_{2_{0}}-\lambda_{0_{1}} \frac{x_{2}}{x_{2}+\lambda_{0}} x_{1} \quad x_{1}(0)=x_{2_{0}} \\
& \dot{x}_{3}=\quad x_{3_{0}} \lambda_{0_{1}} \frac{x_{2}}{x_{2}+\lambda_{0_{2}}}-\lambda_{0_{3}}\left(1-\frac{\lambda_{0_{4}}}{x_{3}}\right) \quad x_{3}(0)=x_{3_{0}} \\
& \dot{x}_{4}=\frac{\lambda_{0_{3}} \lambda_{0_{4}} x_{1} x_{6}}{x_{3}^{2}}+\lambda_{0_{3}} x_{4}\left(1-\frac{\lambda_{0_{4}}}{x_{3}}\right) \quad x_{4}(0)=0 \\
& \dot{x}_{5}=-\frac{x_{1} x_{2}}{x_{2}+\lambda_{0_{2}}}-\frac{\lambda_{0_{1}} x_{2} x_{4}}{\left(x_{2}+\lambda_{0_{2}}\right)}+x_{5}\left(\frac{\lambda_{0_{1}} x_{1} x_{2}}{\left(x_{2}+\lambda_{0_{2}}\right)^{2}}-\frac{\lambda_{0_{1}} x_{1}}{\left(x_{2}+\lambda_{0_{2}}\right)}\right) \quad x_{5}(0)=0 \\
& \dot{x}_{6}=\frac{x_{2}}{x_{2}+\lambda_{0_{2}}}+x_{5}\left(\frac{\lambda_{0_{1}} x_{2}}{\left(x_{2}+\lambda_{0_{2}}\right)^{2}}+\frac{\lambda_{0_{1}}}{\left(x_{2}+\lambda_{0_{2}}\right)}\right)-\lambda_{0_{3}} x_{6} \quad x_{6}(0)=0 \\
& \dot{x}_{7}=\frac{\lambda_{0_{3}} \lambda_{0_{4}} x_{1} x_{9}}{x_{3}^{2}}+\lambda_{0_{3}} x_{7}\left(1-\frac{\lambda_{0_{4}}}{x_{3}}\right) \quad x_{7}(0)=0 \\
& \dot{x}_{8}=\frac{\lambda_{0_{1}} x_{1} x_{2}}{\left(x_{2}+\lambda_{0_{2}}\right)^{2}}-\frac{\lambda_{0_{1}} x_{2} x_{7}}{\left(x_{2}+\lambda_{0_{2}}\right)}+x_{8}\left(\frac{\lambda_{0_{1}} x_{1} x_{2}}{\left(x_{2}+\lambda_{0_{2}}\right)^{2}}-\frac{\lambda_{0_{1}} x_{1}}{\left(x_{2}+\lambda_{0_{2}}\right)}\right) \quad x_{8}(0)=0 \\
& \dot{x}_{9}=-\frac{\lambda_{0_{1}} x_{2}}{\left(x_{2}+\lambda_{0_{2}}\right)^{2}}+x_{8}\left(\frac{\lambda_{0_{1}} x_{2}}{\left(x_{2}+\lambda_{0_{2}}\right)^{2}}+\frac{\lambda_{0_{1}}}{\left(x_{2}+\lambda_{0_{2}}\right)}\right)-\lambda_{0_{3}} x_{9} \quad x_{9}(0)=0 \\
& \dot{x}_{10}=\frac{\lambda_{0_{3}} \lambda_{0_{4}} x_{1} x_{12}}{x_{3}^{2}}+\lambda_{0_{3}} x_{10}\left(1-\frac{\lambda_{0_{4}}}{x_{3}}\right)+x_{1}\left(1-\frac{\lambda_{0_{4}}}{x_{3}}\right) \quad x_{10}(0)=0 \\
& \dot{x}_{11}=-\frac{\lambda_{0_{1}} x_{10} x_{2}}{\left(x_{2}+\lambda_{0_{2}}\right)}+x_{11}\left(\frac{\lambda_{0_{1}} x_{1} x_{2}}{\left(x_{2}+\lambda_{0_{2}}\right)^{2}}-\frac{\lambda_{0_{1}} x_{1}}{\left(x_{2}+\lambda_{0_{2}}\right)}\right) \quad x_{11}(0)=0 \\
& \dot{x}_{12}=x_{11}\left(-\frac{\lambda_{0_{1}} x_{2}}{\left(x_{2}+\lambda_{0_{2}}\right)^{2}}+\frac{\lambda_{0_{1}}}{\left(x_{2}+\lambda_{0_{2}}\right)}\right)-\lambda_{0_{3}} x_{12}-x_{3}\left(1-\frac{\lambda_{0_{4}}}{x_{3}}\right) \quad x_{12}(0)=0 \\
& \dot{x}_{13}=\frac{\lambda_{0_{3}} \lambda_{0_{4}} x_{1} x_{15}}{x_{3}^{2}}-\frac{\lambda_{0_{3}} x_{1}}{x_{3}}+\lambda_{0_{3}} x_{13}\left(1-\frac{\lambda_{0_{4}}}{x_{3}}\right) \quad x_{13}(0)=0 \\
& \dot{x}_{14}=-\frac{\lambda_{0_{1}} x_{13} x_{2}}{\left(x_{2}+\lambda_{0_{2}}\right)}+x_{14}\left(\lambda_{0_{1}} \frac{x_{1} x_{2}}{\left(x_{2}+\lambda_{0_{2}}\right)^{2}}-\frac{\lambda_{0_{1}} x_{1}}{\left(x_{2}+\lambda_{0_{2}}\right)}\right) \quad x_{14}(0)=0 \\
& \dot{x}_{15}=\lambda_{0_{3}}+x_{14}\left(-\frac{\lambda_{0_{1}} x_{2}}{\left(x_{2}+\lambda_{0_{2}}\right)^{2}}+\frac{\lambda_{0_{1}}}{\left(x_{2}+\lambda_{0_{2}}\right)}\right)-\lambda_{0_{3}} x_{15} \quad x_{15}(0)=0
\end{aligned}
$$

The solution of this equation was computed for the initial state $x_{1_{0}}=91.483 \mathrm{mgCL}^{-1}$, $x_{2_{0}}=14.5148 \mathrm{mgNL}^{-1}$, and $x_{3_{0}}=0.03047 \mathrm{mgN}(\mathrm{mgC})^{-1}$ as set in the simulation shown in Figure 6. The nominal parameter values $\lambda_{0_{1}}=0.1 \mathrm{mgN}(\mathrm{mgC})^{-1} \mathrm{~d}^{-1}, \lambda_{0_{2}}=2 \mathrm{mgNL}^{-1}$, $\lambda_{0_{3}}=2 \mathrm{~d}^{-1}$, and $\lambda_{0_{4}}=0.03 \mathrm{mgN}(\mathrm{mgC})^{-1}$ used for the simulation were set based on the literature [48]. Figure 8 shows $x_{4}, x_{7}, x_{10}$, and $x_{13}$, which are the sensitivities of $x_{1}$ with respect to $\rho_{m}, k_{y}, \mu$, and $z c_{0}$, respectively. Figure 9 shows the corresponding quantities of $x_{5}, x_{8}, x_{11}$, and $x_{14}$ for $x_{2}$; Figure 10 shows $x_{6}, x_{9}, x_{12}$, and $x_{15}$ for $x_{3}$. An inspection of these figures shows that the solution is more sensitive to variations in the parameters $\lambda_{2}=k_{y}$ and $\lambda_{4}=z c_{0}$ than to variations in the parameters $\lambda_{1}=\rho_{m}$ and $\lambda_{3}=\mu_{m}$. 


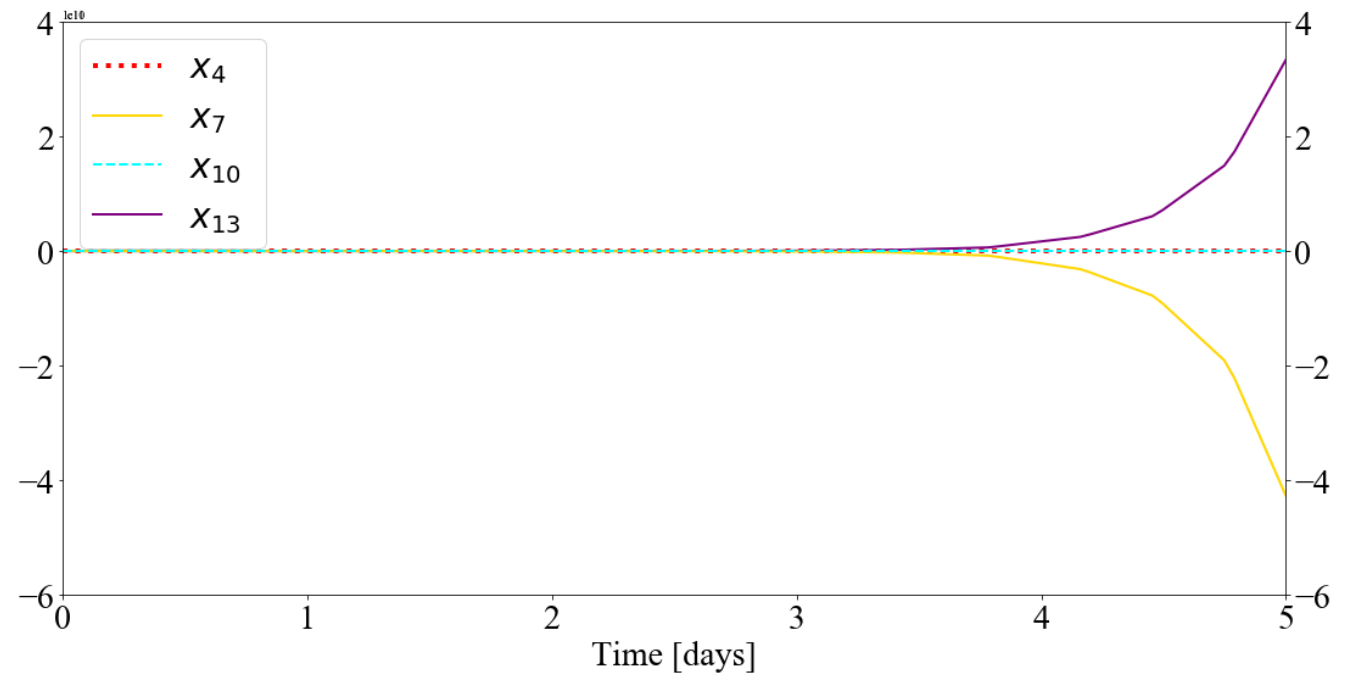

Figure 8. Sensitivity functions $x_{4}, x_{7}, x_{10}$, and $x_{13}$ are the sensitivities $x_{1}$ with respect to $\lambda_{1}, \lambda_{2}, \lambda_{3}$, and $\lambda_{4}$ in time, respectively. The simulation was computed for the initial state $x_{1_{0}}=91.483, x_{2_{0}}=14.5148$, and $x_{3_{0}}=0.03047$. The solution is more sensitive to variations in the parameters $\lambda_{2}=k_{y}$ and $\lambda_{4}=z c_{0}$ than to variations in the parameters $\lambda_{1}=\rho_{m}$ and $\lambda_{3}=\mu_{m}$. We performed the simulation and plotting in Octave 5.1.0.0 and Python 3.7.1, respectively.

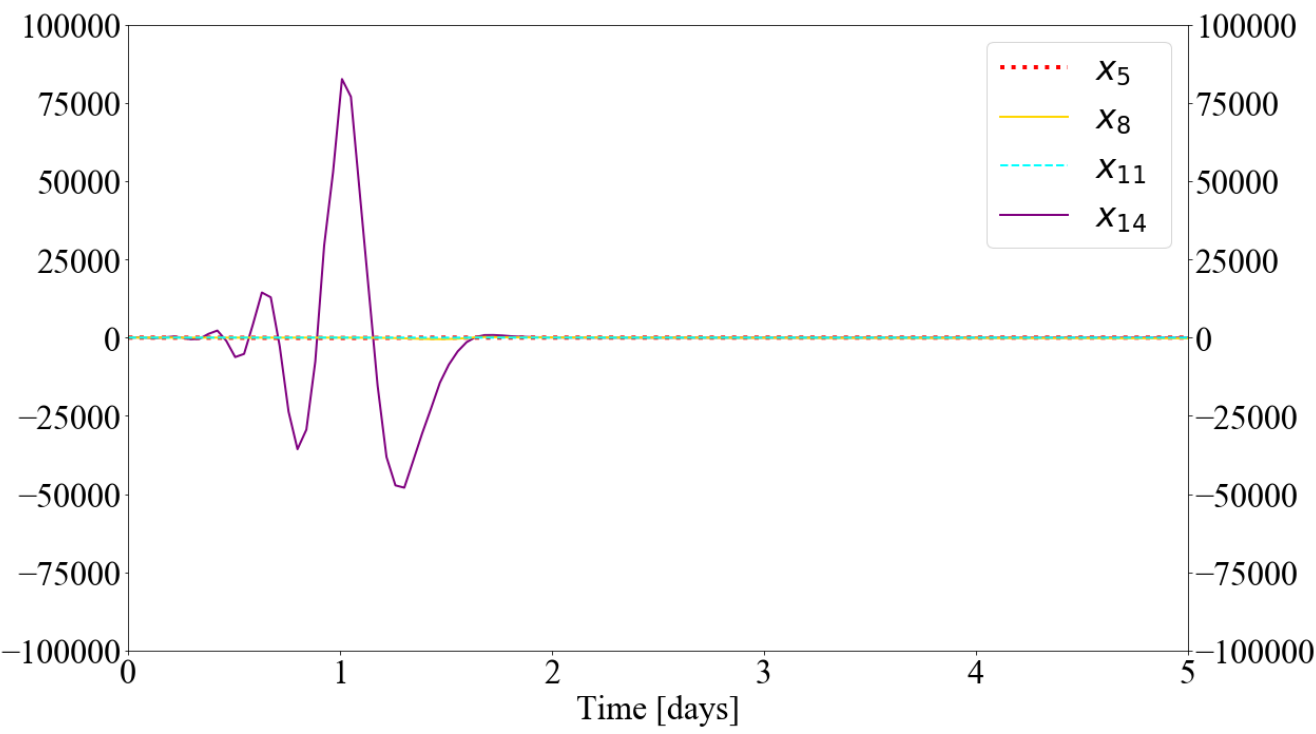

Figure 9. Sensitivity functions $x_{5}, x_{8}, x_{11}$, and $x_{14}$ are the sensitivities $x_{2}$ with respect to $\lambda_{1}, \lambda_{2}, \lambda_{3}$, and $\lambda_{4}$ in time, respectively. The simulation was computed for the initial state $x_{1_{0}}=91.483, x_{2_{0}}=14.5148$, and $x_{3_{0}}=0.03047$. The solution is more sensitive to variations in the parameters $\lambda_{2}=k_{y}$ and $\lambda_{4}=z c_{0}$ than to variations in the parameters $\lambda_{1}=\rho_{m}$ and $\lambda_{3}=\mu_{m}$. We performed the simulation and plotting in Octave 5.1.0.0 and Python 3.7.1, respectively. 


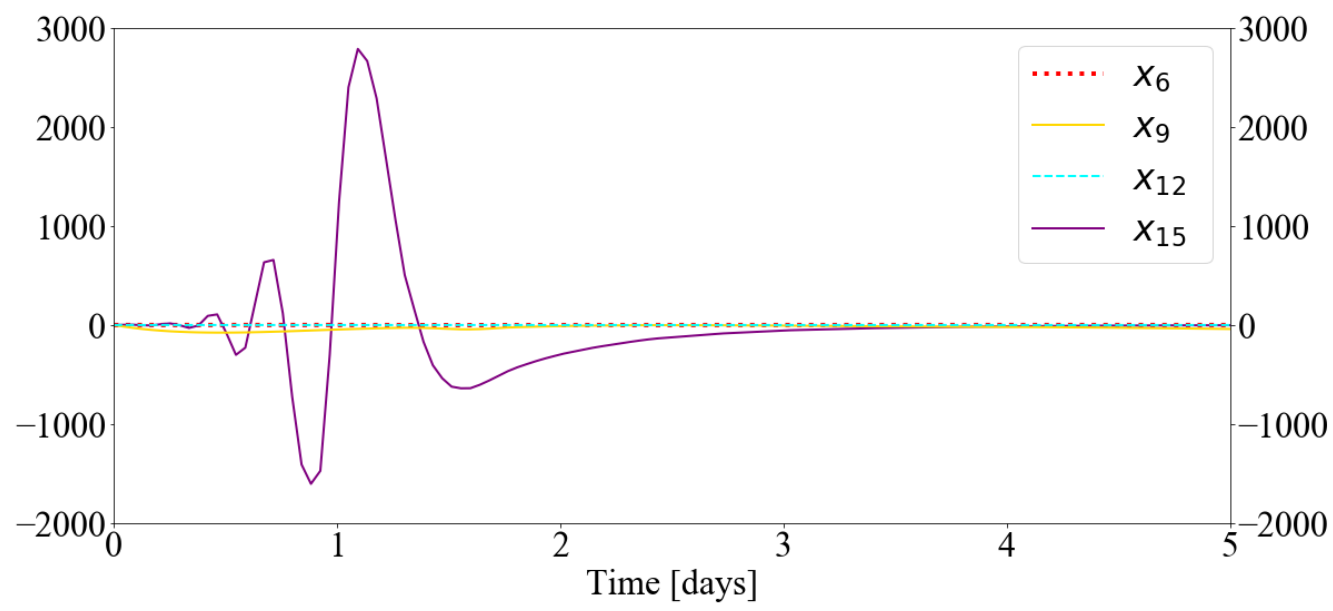

Figure 10. Sensitivity functions $x_{6}, x_{9}, x_{12}$, and $x_{15}$ are the sensitivities $x_{3}$ with respect to $\lambda_{1}, \lambda_{2}$, $\lambda_{3}$, and $\lambda_{4}$ over time, respectively. The simulation was computed for the initial state $x_{1_{0}}=91.483$, $x_{2_{0}}=14.5148$, and $x_{3_{0}}=0.03047$. The solution is more sensitive to variations in the parameters $\lambda_{2}=k_{y}$ and $\lambda_{4}=z c_{0}$ than to variations in the parameters $\lambda_{1}=\rho_{m}$ and $\lambda_{3}=\mu_{m}$. We performed the simulation and plotting in Octave 5.1.0.0 and Python 3.7.1, respectively.

\section{Discussion}

The study of the Droop model suggests how microalgae growth real-processes behave. The Droop model captures some features of the growth physical process. The expression (long-term) used for describing equilibrium $v_{1}$ represents the expected production of a batch culture over a few days and not its average lifespan. At the laboratory scale, batch cultures do not last very long. It is assumed that after a certain time, the growth rate then reaches zero at the stationary phase since nutrients in the culture medium are exhausted. In this phase, the experimental observations no longer match the results predicted by the model. Then, the process is stopped and the storage carbon products in the cells (e.g., starch, and neutral lipid) are harvested. Algal mortality usually plays only a minor role in batch culture systems because the residence time of the cells in the reactor is much shorter than their average lifespan due to the cultivation purposes, which are normally used to reach a stable phase of growth. Therefore, algal mortality was neglected in this batch culture model analysis.

The stability concept of algal systems in real cultures means that the trajectories of the variables of the dynamic system do not present sudden changes under low disturbances in the initial conditions, such as the concentration of nutrients. An unstable behavior in algal growth systems leads to imprecise growth kinetics predictions and biomass production. Changes in the initial conditions are proportional to changes in the equilibrium reached in the steady state.

In nature, few parameters are nearly constant because of the multi-variant conditions; as such, identifying the parameters that have the most impact on the model could provide an idea as to which parameters are more important in parameter calibration tasks. Despite the model seeming to have simple dynamics, where the behavior mainly depends on the initial state conditions of the system, it is important to emphasize how sensitive these solutions are to the variation in the parameters, and to the stage of growth.

The sensitivity analysis of model parameters in this work can be useful for predicting optimal algal growth conditions over time. The proposed sensitivity equations can be simulated with various experimental conditions that may vary with the type of microalgae species, culture media, and photobioreactor configuration, amongst others factors, to identify which parameters may be influenced by other external factors and impact the final results of the state variables over time. 


\section{Conclusions}

This study was dedicated to an in-depth analysis of a microalgal growth model developed by Droop for nutrient-limited batch cultivation. We performed a stability analysis of the equilibria and a detailed analysis of the system stability. Two equilibria were found at the stationary state, representing either the absence of biomass or the longterm biomass production of major physical interest. The long-term biomass production equilibrium is stable. The equilibrium of the absence of biomass was found to be unstable. An examination of the found equilibria showed that the system does not have isolated fixed points; rather, it has an infinite number of equilibria depending on the values of minimal cell quota and initial conditions. The results of the sensitivity analysis indicated that model outputs were especially sensitive to the parameter of half saturation constant $k_{y}$ and minimal cell quota $z c_{0}$ than to variations in the maximum inorganic nutrient absorption rate $\rho_{m}$ and maximum growth rate $\mu_{m}$. Additionally, these results could be useful for understanding the long-term equilibria and stability behavior of this model when far from thermodynamic equilibrium from a more physical and explicit point of view.

Author Contributions: Conceptualization, A.G.-P. and L.A.-V.; methodology, A.G.-P., L.A.-V. and H.H.-E.; software, A.G.-P. and P.M.G.-V.; validation, A.G.-P., L.A.-V., H.H.-E. and P.M.G.-V.; formal analysis, A.G.-P., L.A.-V. and H.H.-E.; investigation, A.G.-P., L.A.-V., H.H.-E. and P.M.G.-V.; resources, A.G.-P., L.A.-V., H.H.-E. and P.M.G.-V.; data curation, A.G.-P., L.A.-V. and P.M.G.-V.; writing-original draft preparation, A.G.-P. and L.A.-V.; writing-review and editing, A.G.-P., L.A.-V., H.H.-E. and P.M.G.-V.; visualization, A.G.-P., L.A.-V., H.H.-E. and P.M.G.-V.; supervision, L.A.-V., H.H.-E. and P.M.G.-V.; project administration, L.A.-V. and A.G.-P.; funding acquisition, L.A.-V. All authors have read and agreed to the published version of the manuscript.

Funding: The first author was funded by Consejo Nacional de Ciencia y Tecnología grant number 724370 .

Institutional Review Board Statement: Not applicable.

Informed Consent Statement: Not applicable.

Data Availability Statement: Not applicable.

Acknowledgments: Abraham Guzmán Palomino gratefully acknowledges the scholarship No. 724370 from CONACyt to pursue his postgraduate studies and the Mexican Institute of Complex Systems for supporting this research.

Conflicts of Interest: The authors declare no conflict of interest.

\section{References}

1. Chen, C.Y.; Zhao, X.; Yen, H.W.; Ho, S.H.; Cheng, C.L.; Lee, D.J.; Bai, F.W.; Chang, J.S. Microalgae-based carbohydrates for biofuel production. Biochem. Eng. J. 2013, 78, 1-10. [CrossRef]

2. Patnaik R.; Mallick, N. Microalgal biodiesel production: realizing the sustainability index. Front. Bioeng. Biotechnol. 2021, 9. [CrossRef] [PubMed]

3. Rizwan, M.; Mujtaba, G.; Memon, S.A.; Lee, K.; Rashid, N. Exploring the potential of microalgae for new biotechnology applications and beyond: A review. Renew. Sustain. Energy Rev. 2018, 92, 394-404. [CrossRef]

4. Fernández I.; Guzmán J.L.; Berenguel M.; Acién F.G. Dynamic Modeling of Microalgal Production in Photobioreactors. In Prospects and Challenges in Algal Biotechnology; Tripathi, B., Kumar, D., Eds.; Springer: Singapore, 2017; pp. $49-89$.

5. Albarello, A.; Simionato, D.; Morosinotto, T.; Bezzo, F. Model-Based Optimization of Microalgae Growth in a Batch Plant. Ind. Eng. Chem. Res. 2019, 58, 5121-5130. [CrossRef]

6. Huang, Q.; Jiang, F.; Wang, L.; Yang, C. Design of Photobioreactors for Mass Cultivation of Photosynthetic Organisms. Engineering 2017, 3, 318-329. [CrossRef]

7. Eze, V.C.; Velasquez-Orta, S.B.; Hernández-García, A.; Monje-Ramírez, I.; Orta-Ledesma, M.T. Kinetic modelling of microalgae cultivation for wastewater treatment and carbon dioxide sequestration. Algal Res. 2018, 32, 131-141. [CrossRef]

8. Rafay, R.; Uratani, J.M.; Hernandez, H.H.; Rodríguez, J.; Monje-Ramírez, I.; Orta-Ledesma, M.T. Growth and Nitrate Uptake in Nannochloropsis gaditana and Tetraselmis chuii Cultures Grown in Sequential Batch Reactors. Front. Mar. Sci. 2020, 7, 1-9. [CrossRef]

9. Posten, C. Microalgae Biotechnology. Front. Mar. Sci. 2016, 153, 59-89. 
10. Al-Qasmi, M.; Raut, N.; Talebi, S.; Al-Rajhi, S.; Al-Barwani, T. A Review of Effect of Light on Microalgae Growth. Proc. World Congr. Eng. 2012, 7, 8-10.

11. Bernard, O.; Mairet, F. A Modelling of Microalgae Culture Systems with Applications to Control and Optimization. Proc. World Congr. Eng. 2015, 153, 59-87._2014_287. [CrossRef]

12. Hsu, S.B.; Ho, Y.H.; Wang, F.B. Mathematical analysis on a droop model with intraguild predation. Taiwan. J. Math. 2019, 23, 351-373. [CrossRef]

13. Lee, E.; Jalalizadeh, M.; Zhang, Q. Growth kinetic models for microalgae cultivation: A review. Algal Res. 2015, 12, 497-512. [CrossRef]

14. Darvehei, P.; Bahri, P.A.; Moheimani, N.R. Model development for the growth of microalgae: A review. Renew. Sustain. Energy Rev. 2021, 9, 233-258. [CrossRef]

15. Park, J.; Jin, H.; Lim, B.; Park, K.; Lee, K. Ammonia removal from anaerobic digestion effluent of livestock waste using green alga Scenedesmus sp. Bioresour. Technol. 2010, 22, 8649-8657. [CrossRef] [PubMed]

16. Andrews, J.F. A mathematical model for the continuous culture of microorganisms utilizing inhibitory substrates. Biotechnol. Bioeng. 1968, 6, 707-723. [CrossRef]

17. Kurano, N.; Miyachi, S. Selection of microalgal growth model for describing specific growth rate-light response using extended information criterion. Biosci. Bioeng. 2005, 4, 403-408. [CrossRef]

18. Martínez Sancho, M.; Jimenez Castillo, J.; El Yousfi, F. Influence of phosphorus concentration on the growth kinetics and stoichiometry of the microalga Scenedesmus obliquus. Process Biochem. 1997, 8, 657-664. [CrossRef]

19. Regaudie-de-Gioux, A.; Sal, S.; López-Urrutia, Á. Poor correlation between phytoplankton community growth rates and nutrient concentration in the sea. Biogeosci. Discuss. 2014, 10, 14797-14818.

20. Flynn, K.J. Modelling multi-nutrient interactions in phytoplankton; balancing simplicity and realism. Prog. Oceanogr. 2003, 56, 249-279. [CrossRef]

21. Singh, D.; Nedbal, L.; Ebenhöh, O. Modelling phosphorus uptake in microalgae. Biochem. Soc. Trans. 2018, 46, 483-490. [CrossRef]

22. Tamiya, H.; Hase, E.; Shibata, K.; Mituya, A.; Iwamura, T.; Nihei, T.; Sasa, T. Kinetics of growth of Chlorella, with special reference to its dependence on quantity of available light and on temperature. In Algal Culture: from Laboratory to Pilot Plant; Burlew, J. S., Ed.; Carnegie Institution of Washington: Washington, DC, USA, 1953; pp. 204-234.

23. Chae, S.; Hwang, E.; Shin, H. Single cell protein production of Euglena gracilis and carbon dioxide fixation in an innovative photo-bioreactor. Bioresour. Technol. 2006, 97, 322-329. [CrossRef]

24. van Oorschot, J.L.P. Conversion of Light Energy in Algal Culture; Wageningen University: Wageningen, The Netherlands, 1955.

25. Bannister, T. Quantitative description of steady state, nutrient-saturated algal growth, including adaptation. Limnol. Oceanogr. 1979, 24, 76-96. [CrossRef]

26. Chalker, B.E. Modeling light saturation curves for photosynthesis: An exponential function, J. Theor. Biol. 1980, 24, 205-215. [CrossRef]

27. Droop, M.R. The nutrient status of algal cells in batch culture. J. Mar. Biol. Assoc. 1974, 54, 825-855. [CrossRef]

28. Flynn, K.J. How critical is the critical n:P ratio? J. Phycol. 2002, 5, 961-970. [CrossRef]

29. Marine, D.; Pa, A. In defence of the Cell Quota model of micro-algal growth. IFAC-PapersOnLine 2003, 25, $103-107$.

30. Flynn, K.J.; Baron, R.; Peavey, D.G. The importance of the form of the quota curve and control of non-limiting nutrient transport in phytoplankton models. J. Plankton Res. 2008, 30, 423-438. [CrossRef]

31. Muñoz-Tamayo, R.; Martinon, P.; Bougaran, G.; Mairet, F.; Bernard, O. Design of optimal 169 experiments for parameter estimation of microalgae growth models. IFAC Proc. 2013, 12, 315-320. [CrossRef]

32. Benvenuti, G.; Bosma, R.; Ji, F.; Lamers, P.; Barbosa, M.J.; Wijels, R.H. Batch and semicontinuous microalgal TAG production in lab-scale and outdoor photobioreactors. J. Appl. Phycol. 2016, 28, 3167-3177. [CrossRef] [PubMed]

33. Lange, K.; Oyarzun, F.J. The attractiveness of the droop equations. Math. Biosci. 1992, 111, 261-278. [CrossRef]

34. Oyarzun, F.J.; Lange, K. The attractiveness of the droop equations II. Generic uptake and growth functions. Math. Biosci. 1994, 121, 127-139. [CrossRef]

35. Clodong, S.; Blasius, B. Chaos in a periodically forced chemostat with algal mortality. Biol. Sci. 2004, 271, 1617-1624. [CrossRef]

36. Datta, B.N. Numerical Methods for Linear Control Systems: Design and Analysis; Elsevier Inc.: Amsterdam, The Netherlands, 2003; Volume 121.

37. Alligood, K.T.; Sauer, T.; Yorke, J.A. Chaos: An Introduction to Dynamical Systems; Springer: Berlin/Heidelberg, Germany, 1996.

38. Li, Z; Xu, R. Stability analysis of a ratio-dependent chemostat model with time delay and variable yield. Int. J. Biomath. 2010, 3, 243-253. [CrossRef]

39. Solimeno, A.; Samsó, R.; García, J. Parameter sensitivity analysis of a mechanistic model to simulate microalgae growth. Algal Res. 2016, 16, 217-223. [CrossRef]

40. Khalil, H. Nonlinear Systems, 2nd ed.; Prentice-Hall: Hoboken, NJ, USA, 1996.

41. Goldman, J.C.; Peavey, D.G. Steady-state growth and chemical composition of the marine chlorophyte Dunaliella tertiolecta in nitrogen-limited continuous cultures. Appl. Environ. Microbiol. 1979, 38, 894-901. [CrossRef]

42. Mairet, F.; Baron, R.; Peavey, D.G. A Physiologically Structured Equation to Consider Quota Heterogeneity in the Droop Model. IFAC-PapersOnLine 2019, 52, 275-280. [CrossRef] 
43. Campolongo, F.; Cariboni, J.; Saltelli, A. An effective screening design for sensitivity analysis of large models. Environ. Model. Softw. 2007, 22, 1509-1518. [CrossRef]

44. Alzahrani, E.O.; El-Dessoky, M.M. Global dynamics of a cell quota-based model of light-dependent algae growth in a chemostat. Commun. Nonlinear Sci. Numer. Simulat. 2020, 90, 105295. [CrossRef]

45. Robinson, C. Stability, Symbolic Dynamics, and Chaos; CRC Press: Boca Raton, FL, USA, 1995.

46. Strogatz, S.H. Nonlinear Dynamics and Chaos; Addison-Wesley: Boston, MA, USA, 1994.

47. Hindmarsh, A.C. ODEPACK, a Systematized Collection of ODE Solvers. In Scientific Computing; North-Holland: Amsterdam, The Netherlands, 1983; pp. 55-64.

48. Benavides, M.; Hantson, A.L.; van Impe, J.; Vande Wouwer, A. Parameter identification of Droop model: an experimental case study. Bioprocess Biosyst. Eng. 2015, 38, 1783-1793. [CrossRef] 